\title{
More on the Triplet Killing Potentials of Quaternionic Kähler manifolds
}

\author{
Shogo Aoyama* \\ Department of Physics \\ Shizuoka University \\ Ohya 836, Shizuoka \\ Japan
}

September 26, 2021

\begin{abstract}
We show the properties of the triplet Killing potentials of quaternionic Kähler manifolds which have been missing in the literature. It is done by means of the metric formula of the manifolds. We compute the triplet Killing potentials for the quaternionic Kähler manifold $S p(n+1) / S p(n) \otimes S p(1)$ as an illustration.
\end{abstract}

PACS: 02.40.ky, 02.40.Tt, 04.65.+e

Keywords: Kähler manifold, Supergravity, Coset space

*e-mail: spsaoya@ipc.shizuoka.ac.jp 
It is well-known that when hypermultiplets couple with $\mathcal{N}=2$ supergravity in four dimensions, the moduli space is required to be a quaternionic Kähler manifold[1, 2]. A quaternionic Kähler manifold is a $4 n$-dimensional real manifold. The holonomy group of quaternionic Kähler manifolds is $S p(1) \otimes S p(n)$ and the $S p(1)$ conection must not vanish. When the gravitational coupling $\kappa \rightarrow 0$, this $S p(1)$ conection is required to vanish, the holonomy group gets contained in $S p(n)$ and the quaternionic Kähler manifold approaches to a hyper Kähler manifold 3. Such a quaternionic or hyper Kähler manifold appears as a moduli space for type II superstring compactification on Calabi-Yau manifolds, together with a special Kähler manifold given by vector multiplets [2]. The direct product of both moduli spaces is an arena for the mirror symmetry in the type II superstring [4].

Quaternionic Kähler manifolds are by now well-studied subjects [1] [5]- 9]. The existence of the triplet Killing potentials $\vec{M}^{A}$ is a hallmark of quaternionic Kähler manifolds when they are group manifolds. The properties of the $\vec{M}^{A}$ were well studied in the literature [7]-9]. But there are still important properties which are missing in generality of the arguments. In this letter we show them, explicitly constructing the metric of quaternionic Kähler manifolds. With the specific metric at hand the geometric quantities of the manifolds like the metric, the Riemann curvature etc, are given in terms of the triplet Killing potentials alone. Among them we stress on the relations implying that $\vec{M}^{A}$ is an eigen vector of the Beltrami-Laplace operator for the manifold and the quantity $\vec{M}^{A} \cdot \vec{M}^{A}$, which was called energy in 9, is a constant related with the Riemann scalar curvature. This is justified as long as the metric of quarternionic Kähler group manifolds manifolds is non-degenerate. Similar relations were known for the Killing potentials which exist for the ordinary Kähler group manifolds 10]-13. We develope our arguments noting a pararellism between both Kähler group manifolds. Finally we compute the triplet Killing potentials for the quarternionic Kähler coset space $S p(n+1) / S p(n) \otimes S p(1)$ to illustrate our general arguments.

We start with a summary of the geometric properties discussed in [7]-9]. A quaternionic Kähler manifold is endowed with a triplet complex structure $\vec{J}_{a}^{b}$. Assume that it is a group manifold admitting an isometry $G$ realized by Killing vectors $R^{A a}$ with $A=1,2, \cdots, \operatorname{dim} G$. For the quantity $\vec{J}_{a}^{b} R_{b}^{A}\left(\equiv \vec{J}_{a}^{b} g_{b c} R^{A c}\right)$ we have triplet Killing vectors $\vec{M}^{A}$ such that

$$
\nabla_{a} \vec{M}^{A}=\nu \vec{J}_{a}^{b} R_{b}^{A}, \quad \nu \equiv \frac{R}{4 n(n+2)},
$$

with the Riemann scalar curvature $R$. It is given by

$$
\vec{M}^{A}=\vec{r}^{A}+R^{A a} \vec{\omega}_{a}
$$

with a spin connection $\vec{\omega}_{a}$ and an appropriate $\vec{r}^{A}$ which will be explained soon later. $\vec{M}^{A}$ can be also written in the form

$$
\vec{M}^{A}=\frac{1}{2 n} \vec{J}_{a}^{b} \nabla_{b} R^{A a}
$$

and satisfies the relation

$$
\nu R_{a}^{A} R_{b}^{B} \vec{J}^{a b}=f^{A B C} \vec{M}^{C}+\vec{M}^{A} \times \vec{M}^{B} .
$$


Here $f^{A B C}$ are the structure constants of $G$ and $\vec{J}^{b c} \equiv g^{b d} \vec{J}_{d}^{c}$.

We remember quite similar arguments for Kähler manifolds[1] [1]. Namely a Kähler manifold is endowed with a singlet complex structure $J_{a}{ }^{b}$. It may be locally set to be

$$
J_{a}^{b}=\left(\begin{array}{cc}
-i \delta_{\alpha}^{\beta} & 0 \\
0 & i \delta_{\bar{\alpha}}^{\bar{\beta}}
\end{array}\right) .
$$

If the Kähler manifold admits an isometry $G$, realized by (anti-)holomorphic Killing vectors $R^{A \alpha}\left(\bar{R}^{A \bar{\alpha}}\right)$, there exist Killing potentials such that

$$
\partial_{\alpha} M^{A}=-i g_{\alpha \bar{\beta}} \bar{R}^{A \bar{\beta}}, \quad \partial_{\bar{\alpha}} M^{A}=i g_{\beta \bar{\alpha}} R^{A \beta} .
$$

It was shown that such Killing potentials are given by

$$
\begin{aligned}
M^{A} & =-\frac{i}{\mathcal{N}_{a d j}} \nabla_{\alpha} R^{A \alpha}\left(=\frac{i}{\mathcal{N}_{a d j}} \nabla_{\bar{\alpha}} \bar{R}^{A \bar{\alpha}}\right), \\
\text { or } \quad M^{A} & =i\left(R^{A \alpha} \partial_{\alpha} K-F^{A}\right),
\end{aligned}
$$

and satisfy the relation

$$
-i R^{B \beta} \bar{R}^{C \bar{\gamma}} g_{\beta \bar{\gamma}}+i R^{C \beta} \bar{R}^{B \bar{\gamma}} g_{\beta \bar{\gamma}}=f^{A B C} M^{A} .
$$

In (6) we have used the normalization $f^{A B C} f^{A B D}=2 \mathcal{N}_{a d j} \delta^{C D}$. In (17) $K$ is the Kähler potential and $F^{A}\left(\bar{F}^{A}\right)$ are (anti-)holomorphic quantities that one may find from the Lievariation

$$
\mathcal{L}_{R^{A}} K=F^{A}+\bar{F}^{A} .
$$

Eqs (11) (41) of quaternionic Kähler group manifolds are so similar to (15) (8) of Kähler group manifolds. About $M^{A}$ of Kähler group manifolds we know more properties than those. By the Lie-variation we have 1 ]

$$
\mathcal{L}_{R^{A}} M^{B}=f^{A B C} M^{C} .
$$

From (51) and (6) it follows that $M^{A}$ is an eigen vector of the Beltrami-Laplace operator as 13

$$
g^{\alpha \bar{\beta}} \nabla_{\alpha} \nabla_{\bar{\beta}} M^{A}=-\mathcal{N}_{a d j} M^{A}
$$

Moreover, when Kähler manifolds are irreducible coset spaces, then the geometric quantities can be written in terms of the Killing potentials 11, 12 ,

$$
\begin{aligned}
g_{\alpha \bar{\beta}} & =\partial_{\alpha} M^{A} \partial_{\bar{\beta}} M^{A} \\
R_{\alpha \bar{\beta} \gamma \bar{\delta}} & =-\partial_{\alpha} \partial_{\bar{\beta}} M^{A} \partial_{\gamma} \partial_{\bar{\delta}} M^{A}=f^{A B E} f^{C D E} R_{\alpha}^{A} R_{\bar{\beta}}^{B} R_{\gamma}^{C} R_{\bar{\delta}}^{D}, \\
R & =-R_{\alpha \bar{\beta} \gamma \bar{\delta}} g^{\alpha \bar{\beta}} g^{\gamma \bar{\delta}}=\mathcal{N}_{a d j}^{2} M^{A} M^{A}
\end{aligned}
$$


The geometrical properties of quaternionic Kähler manifolds which correspond to (10) (14) are missing in the literature[7]-9]. In this letter we show them by completing the pararellism between both Kähler group manifolds. They are

$$
\begin{aligned}
\mathcal{L}_{R^{A}} \vec{M}^{B}= & f^{A B C} \vec{M}^{C}+\vec{r}^{A} \times \vec{M}^{B}, \\
\nabla_{a} \nabla^{a} \vec{M}^{A}= & -2 n \nu \vec{M}^{A}, \\
g_{a b}= & \frac{1}{3 \nu^{2}} \nabla_{a} \vec{M}^{A} \cdot \nabla_{b} \vec{M}^{A} \\
R= & \frac{2}{3} n(n+2) \vec{M}^{A} \cdot \vec{M}^{A}, \\
R_{a b c d}= & \frac{1}{3 \nu^{2}}\left[\left[\nabla_{a}, \nabla_{b}\right], \nabla_{c}\right] \vec{M}^{A} \cdot \nabla_{d} \vec{M}^{A} \\
= & f^{A B E} R_{a}^{A} R_{b}^{B} f^{C D E} R_{c}^{C} R_{d}^{D} \\
& -\frac{1}{2} f^{A B C} R_{a}^{A} R_{b}^{B} R^{C e} f^{D E F} R_{c}^{D} R_{d}^{E} R_{e}^{F} \\
& -\frac{1}{4} f^{A B C} R_{d}^{A} R_{b}^{B} R^{C e} f^{D E F} R_{c}^{D} R_{a}^{E} R_{e}^{F} \\
& +\frac{1}{4} f^{A B C} R_{d}^{A} R_{a}^{B} R^{C e} f^{D E F} R_{c}^{D} R_{b}^{E} R_{e}^{F} .
\end{aligned}
$$

Here $\vec{r}^{A}$ is the same quantity that appeared in (2). These formulae are useful when four-fermi couplings and scalar potentials in $\mathcal{N}=2$ supergravity are analyzed from a phenomelogical point of view, by identifying the isometry group $G$ or some subgroup with a grand unification gauge group.

We give a short review on quaternionic Kähler manifolds. Consider a real $4 n$-dimensional Riemann manifold $\mathcal{M}$ with local coordinates $\phi^{a}=\left(\phi^{1}, \phi^{2}, \cdots, \phi^{4 n}\right)$. The line element of the manifold is given by $d s^{2}=g_{a b} d \phi^{a} d \phi^{b}$. If $\mathcal{M}$ is a quaternionic manifold, there exists a triplet complex structure $\vec{J}_{a}^{b} \equiv\left(J_{a}^{1}{ }^{b}, J_{a}^{2}{ }^{b}, J^{3}{ }_{a}{ }^{b}\right)$ satisfying the property

$$
J_{a}^{\alpha b} J_{b}^{\beta}{ }^{c}=-\delta^{\alpha \beta} \delta_{a}^{c}+\epsilon^{\alpha \beta \gamma} J_{a}^{\gamma c} . \quad \alpha=1,2,3,
$$

and the holonomy group in the tangent space is $S p(1) \otimes S p(n)$. We define a set of vielbein 1 -forms

$$
d \phi^{a} e_{a}^{i \mu}, \quad i=1,2, \quad \mu=1,2, \cdots, 2 n,
$$

which satisfy

$$
e_{a}^{i \mu} e^{b}{ }_{i \mu}=\delta_{a}^{b}, \quad e_{a}^{i \mu} e^{a}{ }_{j \nu}=\delta_{j}^{i} \delta_{\nu}^{\mu} .
$$

The holonomy groups $S p(1)$ and $S p(n)$ act on the indices $i$ and $\mu$ respectively. Using these vielbeins we can construct the triplet complex structure as

$$
\vec{J}_{a}^{b}=-i e_{a}^{i \mu} \vec{\sigma}_{i}^{j} e^{b}{ }_{j \mu} .
$$


Lower or raise the indices as $\vec{J}_{a}^{b} g_{b c} \equiv \vec{J}_{a c}$ and $g^{a b} \vec{J}_{b}^{c}=\vec{J}^{a c}$. Then it is easy to show that $\vec{J}_{a b}=-\vec{J}_{b a}, \vec{J}^{a b}=-\vec{J}^{b a}$. We postulate that the vielbeins are covariantly constant:

$$
\nabla_{a} e_{b}^{i \mu}=\partial_{a} e_{b}^{i \mu}-\Gamma_{a b}^{c} e_{c}^{i \mu}+\omega_{a j \nu}^{i \mu} e_{b}^{j \nu}=0
$$

Here $\omega_{a j \nu}^{i \mu}$ is the spin connection Lie-valued in $S p(1) \otimes S p(n)$, so that

$$
\omega_{a j \nu}^{i \mu}=\omega_{a j}{ }^{i} \delta_{\nu}^{\mu}+\omega_{a \nu}^{\mu} \delta_{j}^{i}
$$

We solve (22) for the spin conections $\omega_{a j}{ }^{i}$ and $\omega_{a \nu}{ }^{\mu}$ to find

$$
\omega_{a j}^{i}=-\frac{1}{2 n} e^{b}{ }_{j \nu} \stackrel{\circ}{\nabla}_{a} e_{b}^{i \nu}, \quad \omega_{a \nu}^{\mu}=-\frac{1}{2} e^{b}{ }_{j \nu} \stackrel{\circ}{\nabla}_{a} e_{b}^{j \mu}
$$

Here $\stackrel{\circ}{\nabla}_{c}$ is the covariant derivative which does not contain the spin connection. From the postulate it follows that the metric and the triplet complex structure are covariantly constant:

$$
\begin{aligned}
\nabla_{a} g_{b c} & =\partial_{a} g_{b c}-\Gamma_{a b}{ }^{e} g_{e c}-\Gamma_{a c}^{e} g_{b e}=0 \\
\nabla_{a}{\overrightarrow{J_{b}}}^{c} & =\partial_{a}{\overrightarrow{J_{b}}}^{c}-\Gamma_{a b}{ }^{e} \vec{J}_{e}^{c}+\Gamma_{a e}{ }^{c} \vec{J}_{b}^{e}+\vec{\omega}_{a} \times{\overrightarrow{J_{b}}}^{c}=0
\end{aligned}
$$

with $\vec{\omega}_{a} \equiv-i \vec{\sigma}_{i}{ }^{j} \omega_{a j}{ }^{i}$. The Riemann curvature tensor is given by

$$
R_{a b c}^{d}=\partial_{b} \Gamma_{a c}^{d}-\partial_{a} \Gamma_{b c}^{d}-\Gamma_{b c}{ }^{e} \Gamma_{a e}^{d}+\Gamma_{a c}{ }^{e} \Gamma_{b e}{ }^{d} .
$$

Correponding to the spin connection (23) it decomposes in the tangent space:

$$
R_{a b c}{ }^{d} e_{d}^{i \mu} e^{c}{ }_{j \nu}=R_{a b j}{ }^{i} \delta_{\nu}^{\mu}+R_{a b \nu}^{\mu} \delta_{j}^{i} .
$$

We may write the $S p(1)$ curveture $R_{a b j}{ }^{i}$ as $\vec{R}_{a b}=-i \vec{\sigma}_{i}{ }^{j} R_{a b j}{ }^{i}$, or equivalently as

$$
\vec{R}_{a b}=-\frac{1}{2 n} R_{a b c}{ }^{d} \vec{J}_{d}^{c}=\frac{1}{2 n} R_{a b c d} \vec{J}^{c d}=\frac{1}{n} R_{c a b}{ }^{d} \vec{J}_{d}^{c} .
$$

From the postulate (22) it is given by

$$
\vec{R}_{a b}=\partial_{[a} \vec{\omega}_{b]}+\vec{\omega}_{a} \times \vec{\omega}_{b}
$$

In [8] they have shown various relations among $\vec{R}_{a b}, \vec{J}_{a}^{b}, R_{a b}\left(\equiv-R_{c a b d} g^{c d}\right)$ and $R(\equiv$ $\left.g^{a b} R_{a b}\right)$ :

$$
\begin{aligned}
R_{a b} & =-\frac{1}{3}(n+2) \vec{J}_{a}^{c} \cdot \vec{R}_{c b}, \\
\vec{R}_{a b} & =\frac{1}{n+2} \vec{J}_{a}^{c} R_{c b}=\frac{1}{2} \vec{J}_{a}^{c} \times \vec{R}_{c b}, \\
R_{a b} & =\frac{1}{4 n} R g_{a b} .
\end{aligned}
$$


Combining (29) and (301) gives

$$
\vec{R}_{a b}=\nu \vec{J}_{a b} .
$$

When the quaternionic Kähler manifold is a coset space $G / H$, there is a set of the Killing vectors $R^{A a}=\left(R^{1 a}, R^{2 a}, \cdots, R^{N a}\right)$ with $N=\operatorname{dim} G$ which represents the isometry group $G$. They are required to satisfy

$$
\begin{aligned}
\mathcal{L}_{R^{A}} R^{B a} & \equiv R^{A b} \partial_{b} R^{B a}-R^{B b} \partial_{b} R^{A a}=f^{A B C} R^{C a}, \\
\mathcal{L}_{R^{A}} g_{a b} & \equiv R^{A c} \partial_{c} g_{a b}+\partial_{a} R^{A c} g_{c b}+\partial_{b} R^{A c} g_{c a}=0, \\
\mathcal{L}_{R^{A}} \vec{J}_{a}^{b} & \equiv R^{A c} \partial_{c} \vec{J}_{a}^{b}+\partial_{a} R^{A c} \vec{J}_{c}{ }^{b}-\partial_{c} R^{A b} \vec{J}_{a}^{c}=\vec{r}^{A} \times \vec{J}_{a}^{b},
\end{aligned}
$$

in which $\mathcal{L}_{R^{A}}$ is the Lie-variation with respect to $R^{A}$. For covariant derivatives of the Killing vectors we have

$$
\begin{aligned}
\nabla_{a} R_{b}^{A}+\nabla_{b} R_{a}^{A} & =0 \\
\nabla_{a} \nabla_{b} R_{c}^{A} & =-R_{b c a}{ }^{d} R_{d}^{A}, \\
\nabla_{a} \nabla_{b} R_{c}^{A}+\nabla_{b} \nabla_{c} R_{a}^{A}+\nabla_{c} \nabla_{a} R_{b}^{A} & =0 . \\
R_{a}^{A} \nabla_{b} R_{c}^{A} & =-\frac{1}{2} f^{A B C} R_{a}^{A} R_{b}^{B} R_{c}^{C},
\end{aligned}
$$

Eq. (35) is the Killing equation which is equivalent to (33). Eq. (36) follows by calculating the l.h.s. as

$$
\begin{aligned}
\nabla_{a} \nabla_{b} R_{c}^{A} & =\frac{1}{2} \nabla_{\{a} \nabla_{b\}} R_{c}^{A}+\frac{1}{2} \nabla_{[a} \nabla_{b]} R_{c}^{A} \\
& =\frac{1}{2}\left(-\nabla_{[a} \nabla_{c]} R_{b}^{A}-\nabla_{[b} \nabla_{c]} R_{a}^{A}\right)+\frac{1}{2} \nabla_{[a} \nabla_{b]} R_{c}^{A} \\
& =\frac{1}{2}\left(-R_{a c b}{ }^{d}-R_{b c a}{ }^{d}+R_{a b c}{ }^{d}\right) R_{d}^{A},
\end{aligned}
$$

with (35) and the cyclic property of the Riemann curvature tensor

$$
R_{a b c}{ }^{d}+R_{b c a}{ }^{d}+R_{c a b}{ }^{d}=0 .
$$

Combining (36) and (39) we obtain (37). When the coset space $G / H$ is irreducible, it follows from (33) or (35) that

$$
g_{a b}=R_{a}^{A} R_{b}^{A} .
$$

In using this metric formula our arguments differ from those in the literature. We will be able to explore more on the triplet Killing potentials $\vec{M}^{A}$. (See the later arguments for the reducible case, if there exists any quaternionic Kähler manifold for this case.) Put (32) in the covariant form

$$
R^{A b} \nabla_{b} R_{c}^{B}-R^{B b} \nabla_{b} R_{c}^{A}=f^{A B C} R_{c}^{C} .
$$


Contract this equation with $R_{a}^{A} R_{b}^{B}$. Using the formula (40) and its consequence $\nabla_{c}\left(R_{a}^{A}\right.$ $\left.R_{b}^{A}\right)=0$ we then get (38).

Now we proceed to derive $(15) \sim(19)$. We calculate the following the Lie-derivative

$$
\mathcal{L}_{R^{A}} \nabla_{c} R^{B b}=R^{A a} \nabla_{a} \nabla_{c} R^{B b}-\nabla_{a} R^{A b} \nabla_{c} R^{B a}+\nabla_{c} R^{A a} \nabla_{a} R^{B b} .
$$

On the other hand we have

$$
\nabla_{c} R^{A a} \nabla_{a} R^{B b}+R^{A a} \nabla_{c} \nabla_{a} R^{B b}-(A \rightleftharpoons B)=f^{A B C} \nabla_{c} R^{B b}
$$

from (32). Eliminating the last two terms in (42) by this formula and using (36) and (39) yields

$$
\begin{aligned}
\mathcal{L}_{R^{A}} \nabla_{c} R^{B b} & =R^{A a} \nabla_{[a} \nabla_{c]} R^{B b}+R^{B a} \nabla_{c} \nabla_{a} R^{A b}+f^{A B C} \nabla_{c} R^{B b} \\
& =f^{A B C} \nabla_{c} R^{B b} .
\end{aligned}
$$

With this and (34) the triplet Killing potentials (3) satisfy the property (15)). Note that

$$
\vec{M}^{A}=-\frac{1}{2 n} \vec{J}^{a b} \nabla_{a} R_{b}^{A}=-\frac{1}{2 n} \nabla_{a}\left(\vec{J}^{a b} R_{b}^{A}\right)=-\frac{1}{2 n \nu} \nabla_{b} \nabla^{b} \vec{M}^{A},
$$

by (11), (31) and (25). This implies that $\vec{M}^{A}$ is an eigen vector of the Beltrami-Laplace operator, i.e. (16). Note also that

$$
\nabla_{a} \vec{M}^{A} \cdot \nabla_{b} \vec{M}^{A}=\nu^{2} \vec{J}_{a}^{c} R_{c}^{A} \cdot \vec{J}_{b}^{d} R_{d}^{A}
$$

by (11). Calculate the r.h.s. with (20). Then (44) becomes (17) owing to (40). Eq. (18) can be shown by manipulating the formula

$$
\vec{J}^{a b} \cdot \vec{J}^{c d} R_{a b c d}=-\vec{J}^{a b} \cdot \vec{J}^{c d} R_{d}^{A} \nabla_{c} \nabla_{a} R_{b}^{A},
$$

which is obvious from (36). By (11) and (3) the r.h.s. becomes

$$
\begin{aligned}
-\frac{\nu}{2 n} \vec{J}^{a b} \cdot \vec{J}^{c d} R_{d}^{A} \nabla_{c} \nabla_{a} R_{b}^{A} & =\nabla^{c} \vec{M}^{A} \cdot \nabla_{c} \vec{M}^{A} \\
& =\nabla^{c}\left[\partial_{c}\left(\frac{1}{2} \vec{M}^{A} \vec{M}^{A}\right)\right]-\vec{M}^{A} \cdot \nabla^{c} \nabla_{c} \vec{M}^{A} .
\end{aligned}
$$

From the property (15) we have $\mathcal{L}_{R^{B}}\left(\vec{M}^{A} \cdot \vec{M}^{A}\right)=0$. Contracting this equation with $R^{B b}$ and using (40) yields $g^{b a} \partial_{a}\left(\vec{M}^{A} \cdot \vec{M}^{A}\right)=0$. If the metric is non-degenerate, we get $\vec{M}^{A} \cdot \vec{M}^{A}$ to be a constant. Then (46) becomes

$$
\vec{J}^{a b} \cdot \vec{J}^{c d} R_{d}^{A} \nabla_{c} \nabla_{a} R_{b}^{A}=-4 n^{2} \vec{M}^{A} \cdot \vec{M}^{A},
$$

by (43). On the other hand the l.h.s. of (45) is calculated as

$$
\vec{J}^{a b} \cdot \vec{J}^{c d} R_{a b c d}=2 n \vec{J}^{a b} \cdot \vec{R}_{a b}=\frac{6 n}{n+2} R,
$$


by (20), (26) and (31). With this and (47) the formula (45) becomes (18). Finally we show (19). It consists of the two equalities. To show the equality in the first line we rewrite formula

$$
\nabla_{[a} \nabla_{b]} \vec{J}_{c d}+\nabla_{[c} \nabla_{d]} \vec{J}_{a b}=0
$$

which is obvious from (25) as

$$
R_{a b c e} \vec{J}_{d}^{e}+R_{a b e d} \vec{J}_{c}^{e}+R_{a e c d} \vec{J}_{b}^{e}+R_{e b c d} \vec{J}_{a}^{e}=0
$$

by (27) and (31). Multiply both sides of (48) by $\vec{J}_{f}^{d}$. Using (201) and (36) we get

$$
-3 R_{a b c f}=R_{d}^{A} \nabla_{e} \nabla_{a} R_{b}^{A} \vec{J}_{c}^{e} \cdot \vec{J}_{f}^{d}+R_{d}^{A} \nabla_{c} \nabla_{a} R_{e}^{A} \vec{J}_{b}^{e} \cdot \vec{J}_{f}^{d}+R_{d}^{A} \nabla_{c} \nabla_{e} R_{b}^{A} \vec{J}_{a}^{e} \cdot \vec{J}_{f}^{d} .
$$

This becomes

$$
-3 R_{a b c f}=-R_{d}^{A} \nabla_{[a} \nabla_{b]} R_{e}^{A} \vec{J}_{c}^{e} \cdot \vec{J}_{f}^{d}+R_{d}^{A} \nabla_{c} \nabla_{a} R_{e}^{A}{\overrightarrow{J_{b}}}^{e} \cdot{\overrightarrow{J_{f}}}^{d}-R_{d}^{A} \nabla_{c} \nabla_{b} R_{e}^{A}{\overrightarrow{J_{a}}}_{a}^{e} \cdot{\overrightarrow{J_{f}}}^{d},
$$

by (35) and (37). Using (11) in the r.h.s. of the last equation leads us to see that the equality in the first line of (19) indeed holds. To show the second equality in (19) we write the Riemann curvature tensor in the form

$$
R_{a b c d}=-\nabla_{c}\left(R_{d}^{A} \nabla_{a} R_{b}^{A}\right)+\left(\nabla_{c} R_{d}^{A}\right)\left(\nabla_{a} R_{b}^{A}\right),
$$

by (36). Calculate the first term of the r.h.s. by (38). We then see that $R_{a b c d}$ is expressed in terms of $\nabla_{b} R_{c}^{A}$ alone. Note the formula

$$
\nabla_{b} R_{c}^{A}=-f^{A B C} R_{b}^{B} R_{c}^{C}+\frac{1}{2} R^{A a} f_{a b c}, \quad f_{a b c} \equiv f^{A B C} R_{a}^{A} R_{b}^{B} R_{c}^{C},
$$

which can be shown by contracting (41) with $R_{b}^{B}$ and using (38) and (40). By this formula and the Jacobi identity for the structure constants $f^{A B C}$, the Riemann curvature tensor (49) gets expressed as given in (19).

We illustrate our general arguments for quaternionic Kähler group manifolds, taking the coset space $S p(n+1) / S p(n) \otimes S p(1)[6]$ as an example. The isometry group $S p(n+1)$ is generated by operators $X^{I J}\left(=X^{J I}\right), I, J=1,2, \cdots, 2(n+1)$, satisfying the Lie-algebra

$$
\left[X^{I J}, X^{K L}\right]=\epsilon^{I K} X^{J L}+\epsilon^{J L} X^{I K}+\epsilon^{I L} X^{J K}+\epsilon^{J K} X^{I L} .
$$

Here $\epsilon^{I J}$ is a constant anti-symmetric tensor, which we take to be

$$
\epsilon^{I J}=-\epsilon^{J I}=-\epsilon_{I J}=\epsilon_{J I}=\left(\begin{array}{ccccc}
0 & 1 & & & 0 \\
-1 & 0 & & & \\
& & \ddots & & \\
0 & & & 0 & 1 \\
0 & & & -1 & 0
\end{array}\right)
$$


for convenience. The generators are decomposed as

$$
\left\{X^{I J}\right\}=\left\{X^{i \mu}, X^{\mu \nu}, X^{i j}\right\}, \quad \mu, \nu=1,2, \cdots, 2 n, \quad i, j=1,2,
$$

in which $X^{\mu \nu}$ and $X^{i j}$ are generators of the homogeneous group $S p(n+1) \otimes S p(1)$ and $X^{i \mu}\left(=X^{\mu i}\right)$ are broken generators. The quadratic Casimir takes the form

$$
\frac{1}{2} X^{I J} X^{K L} \epsilon_{I K} \epsilon_{J L}=X^{i \mu} X^{j \nu} \epsilon_{i j} \epsilon_{\mu \nu}+\frac{1}{2} X^{\mu \nu} X^{\rho \sigma} \epsilon_{\mu \rho} \epsilon_{\nu \sigma}+\frac{1}{2} X^{i j} X^{k l} \epsilon_{i k} \epsilon_{j l} .
$$

The coset space $S p(n+1) / S p(n) \otimes S p(1)$ is locally parametrized by coordinates $\phi^{\boldsymbol{i} \boldsymbol{\mu}}$ corresponding to the broken generators $X^{i \mu}$. The line element is given by

$$
d s^{2}=g_{i \mu, j \nu} d \phi^{i \mu} d \phi^{j \nu} .
$$

We find the Killing vectors as non-linear realization of the Lie-algebra (32):

$$
\begin{aligned}
R^{(k \sigma) i \boldsymbol{\mu}} & \equiv\left[X^{k \sigma}, \phi^{i \boldsymbol{\mu}}\right]=\epsilon^{k \boldsymbol{i}} \epsilon^{\sigma \boldsymbol{\mu}}+\phi^{k \boldsymbol{\mu}} \phi^{i \sigma}, \\
R^{(\rho \sigma) \boldsymbol{i} \boldsymbol{\mu}} & \equiv\left[X^{\rho \sigma}, \phi^{i \boldsymbol{\mu}}\right]=\epsilon^{\rho \boldsymbol{\mu}} \phi^{\boldsymbol{i} \sigma}+\epsilon^{\sigma \boldsymbol{\mu}} \phi^{i \rho}, \\
R^{(k l) i \boldsymbol{\mu}} & \equiv\left[X^{k l}, \phi^{\boldsymbol{i \mu}}\right]=\epsilon^{k i} \phi^{l \boldsymbol{\mu}}+\epsilon^{l i} \phi^{k \boldsymbol{\mu}} .
\end{aligned}
$$

Then the metric is obtained from (40)

$$
\begin{aligned}
& g^{i \boldsymbol{\mu}, \boldsymbol{j} \boldsymbol{\nu}}=[\epsilon(1-\epsilon \phi \epsilon \phi)]^{i j}[\epsilon(1-\epsilon \phi \epsilon \phi)]^{\boldsymbol{\mu} \nu}, \\
& g_{\boldsymbol{i \mu}, \boldsymbol{j} \boldsymbol{\nu}}=\left[(1-\epsilon \phi \epsilon \phi)^{-1} \epsilon\right]_{i \boldsymbol{j}}\left[(1-\epsilon \phi \epsilon \phi)^{-1} \epsilon\right]_{\boldsymbol{\mu \nu}} .
\end{aligned}
$$

Here one should undestand matrix multiplication such that

$$
\begin{aligned}
& (\epsilon \phi)_{i}{ }^{\mu}=\epsilon_{\boldsymbol{i k}} \phi^{\boldsymbol{k} \mu}, \quad(\epsilon \phi)_{\mu}{ }^{\boldsymbol{i}}=\epsilon_{\boldsymbol{\mu} \rho} \phi^{\boldsymbol{i \mu}}, \\
& (\epsilon \phi \epsilon \phi)_{i}^{j}=\epsilon_{i \boldsymbol{k}} \phi^{\boldsymbol{k} \rho} \epsilon_{\boldsymbol{\rho} \boldsymbol{\sigma}} \phi^{j \sigma}, \quad(\epsilon \phi \epsilon \phi)_{\mu}^{\nu}=\epsilon_{\boldsymbol{\mu} \boldsymbol{\rho}} \phi^{\boldsymbol{k} \rho} \epsilon_{\boldsymbol{k l}} \phi^{\boldsymbol{l}} .
\end{aligned}
$$

We calculate the Affine connection as

$$
\Gamma_{\boldsymbol{i} \boldsymbol{\mu}, \boldsymbol{j} \boldsymbol{\nu}}^{\boldsymbol{k} \boldsymbol{\lambda}}=\delta_{\boldsymbol{j}}^{\boldsymbol{k}} \delta_{\boldsymbol{\mu}}^{\boldsymbol{\lambda}}[(1-\epsilon \phi \epsilon \phi) \epsilon \phi \epsilon]_{i \boldsymbol{\nu}}+\delta_{\boldsymbol{i}}^{\boldsymbol{k}} \delta_{\nu}^{\boldsymbol{\lambda}}[(1-\epsilon \phi \epsilon \phi) \epsilon \phi \epsilon]_{\boldsymbol{j} \boldsymbol{\mu}} .
$$

The vielbeins are given by

$$
e_{i \boldsymbol{\mu}}^{i \mu}=\left[(1-\epsilon \phi \epsilon \phi)^{-\frac{1}{2}}\right]_{i}^{i}\left[(1-\epsilon \phi \epsilon \phi)^{-\frac{1}{2}}\right]_{\boldsymbol{\mu}}^{\mu},
$$

so as to satisfy

$$
g_{\boldsymbol{i \mu}, \boldsymbol{j} \nu}=e_{i \boldsymbol{\mu}}^{i \mu} e_{j \nu}^{j \nu} \epsilon_{i j} \epsilon_{\mu \nu}
$$

Using the formula

$$
(\phi \epsilon \phi)^{i j}=\phi^{i \boldsymbol{\rho}} \epsilon_{\boldsymbol{\rho} \boldsymbol{\sigma}} \phi^{j \sigma}=\frac{1}{2} \epsilon^{i j} \phi^{2},
$$

with $\phi^{2}=\epsilon_{\boldsymbol{j} \boldsymbol{i}} \phi^{\boldsymbol{i} \boldsymbol{\rho}} \epsilon_{\boldsymbol{\rho} \boldsymbol{\sigma}} \phi^{\boldsymbol{j} \boldsymbol{\sigma}}$, we rewrite the vielbeins in the form

$$
e_{\boldsymbol{i} \boldsymbol{\mu}}^{i \mu}=\delta_{\boldsymbol{i}}^{i}[(1-\epsilon \phi \epsilon \phi)]_{\boldsymbol{\mu}}^{\mu}\left(1-\frac{1}{2} \phi^{2}\right)^{-\frac{1}{2}} .
$$


Then the triplet complex structure (21) becomes

$$
\vec{J}_{i \mu}^{j \nu}=-i \vec{\sigma}_{i}^{j} \delta_{\mu}^{\nu}
$$

Using (3) with (50), (151) and (52) we calculate the triplet Killing potentials to find

$$
\vec{M}^{k \sigma}=-2 i \frac{(\phi \vec{\sigma})^{\rho k}}{1-\frac{1}{2} \phi^{2}}, \quad \vec{M}^{\rho \sigma}=2 i \frac{(\phi \vec{\sigma} \epsilon \phi)^{\rho \sigma}}{1-\frac{1}{2} \phi^{2}}, \quad \vec{M}^{k l}=-2 i \frac{(\epsilon \vec{\sigma})^{k l}}{1-\frac{1}{2} \phi^{2}}
$$

The Casimir product of $\vec{M}^{A}$ is indeed constant:

$$
\vec{M}^{I J} \vec{M}^{K L} \epsilon_{I K} \epsilon_{J L}=12 .
$$

It is easy to check that the triplet Killing potentials have the property (15) with

$$
\vec{r}^{k \sigma}=-i(\phi \vec{\sigma})^{k \sigma}, \quad \vec{r}^{\rho \sigma}=0, \quad \vec{r}^{k l}=-2 i(\epsilon \vec{\sigma})^{k l} .
$$

On the other hand these $\vec{r}$ s may be obtained from (34) as well. Of course they coincide with each other.

The derivations of (17) $\sim(19)$ relied on the metric formula (40). This formula holds at least for a class of quaternionic Kähler coset spaces, called the Wolf space [5], for which $S p(n+1) / S p(n) \otimes S p(1)$ is the simplest example. To verify this we use the CCWZ formalism[15]. The Wolf space is a compact coset space of type $G / S \otimes S p(1)$ or $G / S \otimes$ $S U(2)$ with particularly chosen $G$ and $S$. The generators of $G$, denoted by $T^{A}$, are decomposed as

$$
\begin{gathered}
\left\{T^{A}\right\}=\left\{X^{i \mu}, S^{I}, Q^{\alpha}\right\}, \\
i=1,2, \quad \mu=1,2, \cdots 2 n, \quad I=1,2, \cdots, \operatorname{dim} K, \quad \alpha=1,2,3 .
\end{gathered}
$$

Here $S^{I}$ are generators of the homogeneous group $S$, while $Q^{\alpha}$ those of $S p(1)$ or $S U(2)$ depending on the type. $X^{i \mu}$ are broken generators and transform as a tensor in the representation $(\underline{2}, \underline{2 n})$ under the homogeneous group $S p(1) \otimes S$ or $S U(2) \otimes S$. In general it is said that the coset space is irreducible(reducible) when the representation of $X^{i \mu}$ is irreducible(reducible) under $S$. The Wolf space is irreducible. The quadratic Casimir of $G$ is given by

$$
T^{A} T^{A}=X^{i \mu} X^{j \nu} \eta_{i \mu, j \nu}+S^{I} S^{I}+Q^{\alpha} Q^{\alpha}
$$

in which $\eta_{i \mu, j \nu}$ is the Killing form for the coset part, i.e., $\eta_{i \mu, j \nu}=\epsilon_{i j} \epsilon_{\mu \nu}$ for the previous example $S p(n+1) / S p(n) \otimes S p(1)$. The coset space is locally parametrized by $4 n$ real coordinates $\phi^{i \boldsymbol{\mu}}$ corresponding to the broken generators $X^{i \mu}$. Cosider the quantity $U=\exp \left(\phi^{i \boldsymbol{\mu}} X^{j \nu} \eta_{\boldsymbol{i} \boldsymbol{\mu}, j \nu}\right)$. By left multiplication of $\exp \left(\epsilon^{A} T^{A}\right) \in G$ we find

$$
\exp \left(\epsilon^{A} T^{A}\right) U(\phi)=U\left(\phi^{\prime}\right) h(\phi, g)
$$


appropriately choosing the conpensator $h(\phi, g) \in S p(1) \otimes S$ or $S U(2) \otimes S$. Here $\epsilon^{A}$ are global parameters. When they are infinitesimal (53) yields the Killing vectors $R^{A i \mu}(\phi)$ as

$$
\delta \phi^{i \mu}=\phi^{i \mu}-\phi^{i \mu}=\epsilon^{A} R^{A i \mu}(\phi) .
$$

The fundamental object to construct the metric is the Cartan-Maurer 1-form $U^{-1} d U$. It is valued in the Lie-algebra of $G$ as

$$
U^{-1} d U=e^{i \mu} X^{i \mu} \eta_{i \mu, j \nu}+\omega^{I} S^{I}+\omega^{\alpha} Q^{\alpha} .
$$

$e^{i \mu}$ is a vielbein 1-form, while $\omega^{I}$ and $\omega^{\alpha}$ connection 1-forms corresponding to the respective holonomy groups $S$ and $S p(1)$. With this vielbein 1-form the metric $g_{\boldsymbol{i} \boldsymbol{\mu}, \boldsymbol{j} \boldsymbol{\nu}}$ is given by

$$
g_{\boldsymbol{i \mu}, \boldsymbol{j \nu}}=e_{\boldsymbol{i \mu}}^{i \mu} e_{\boldsymbol{j} \boldsymbol{\nu}}^{j \nu} \eta_{i \mu, j \nu} .
$$

It is also given by (401) i.e., $g_{\boldsymbol{i \mu}, j \boldsymbol{\nu}}=R_{\boldsymbol{i} \boldsymbol{\mu}}^{A} R_{j \boldsymbol{\nu}}^{A}$. Both metrics are equivalent because the value at the origin $\left.g_{\boldsymbol{i \mu}, j \boldsymbol{\nu}}\right|_{\phi=0}$ and the Lie-variation with respect to the Killing vectors $\mathcal{L}_{R^{A}} g_{\boldsymbol{i} \boldsymbol{\mu}, \boldsymbol{j} \boldsymbol{\nu}}$ are the same. Equivalently we can say that

$$
e_{\boldsymbol{i \mu}}^{i \mu}=R_{\boldsymbol{i \mu}}^{A} U^{A, i \mu},
$$

in which $U^{A, i \mu}$ are matrix elements of $U$ in the adjoint representation of $G[12$. The point is that the Wolf space is irreducible. For reducible coset spaces the metric formula (54) should be generalized as

$$
g_{\boldsymbol{i} \boldsymbol{\mu}, \boldsymbol{j} \boldsymbol{\nu}}=\sum_{\mu=1}^{N} c_{\mu} e_{\boldsymbol{i} \boldsymbol{\mu}}^{i \mu} e_{\boldsymbol{j} \boldsymbol{j}}^{j \nu} \eta_{i \mu, j \nu},
$$

in which $c_{\mu}$ may take different values for each irreducible component of $X^{i \mu}$ such as $c_{\mu}=\left(c_{1}, \cdots, c_{1}, c_{2}, \cdots, c_{2}, c_{3} \cdots \cdots, c_{N}, \cdots, c_{N}\right)$ with $\sum_{\mu=1}^{N} 1=2 n$ [12, 14]. Accordingly the formula (40) is generalized to the one obtained by putting the vielbeins (55) in (56) 12. Presumably quaternionic Kähler manifolds could exist also in such reducible cases similarly to the ordinary Kähler manifold [12, 14].

In this letter quaternionic Kähler manifolds were studied in view of an explicit construction of the metric. For the triplet Killing potentials we have shown the properties (15) (19) which have been missing in the literature. Among them the properties (17) (19) were derived with recourse to the metric formula (40). It seems that those properties have been overlooked in generality of the arguments with no specification of the metric. The metric can not be determined by the Killing equation (35) alone. One needs the initial condition at the origin of the manifold. By taking account of it the metric formula (40) was justified for the irreducible coset space. It is worth studying also the reducible case in the constructive approach of this letter.

\section{Acknowledgements}


I thank R. D'Auria for the hospitality at Politecnico di Torino. I also thank him and A. Van Proeyen for the discussions during the stay through which this work was started. The work was supported in part by the Grant-in-Aid for Scientific Research No. 13135212.

\section{References}

[1] J. Bagger and E. Witten, "Matter cupplings in $N=2$ supergravity", Nucl. Phys. B222(1983)1.

[2] B. de Wit, P.G. Lauwers and A. Van Proeyen, "Lagrangians of $N=2$ supergravitymatter systems", Nucl. Phys. B255(1985)569.

[3] L. Alvarez-Gaume and D.Z. Freedman, "Ricci-flat Kähler manifolds and supersymmetry", Phys Lett. B94(1980)171.

[4] N. Seiberg, "Observations on the moduli space of superconformal field theories", Nucl. Phys. B303(1988)286;

S. Cecotti, S. Ferrara and L. Girardello, "Geometry of type II superstrings and the moduli of superconformal field theories", Int. J. Mod. Phys. A4(1989)2475;

P. Candelas and X.C. de la Ossa, "Moduli space of Calabi-Yau manifolds, Nucl. Phys. B355(1991)455.

[5] J.A. Wolf, "Complex homogeneous contact manifolds and quaternionic symmetric spaces", J. Math. Mech. 14(1965)1033.

S. Ishihara, "Quaternion Kählerian manifolds", J. Diff. Geom. 9(1974)483;

D.V. Alekseevsky, "Classification of quaternionic spaces with a transitive soluvable group of motions", Math. USSR Izv. 9(1975)297;

D. Joyce, "Compact hypercomplex and quaternionic manifolds", J. Diff. Geom. $35(1992) 743$.

[6] S. Aoyama and J.W. van Holten, " $\sigma$-models on quaternionic manifolds and anomalies", Z. Phys. C31(1986)487.

[7] G. Sierra and P.K. Townsend, "The gauge invariant $N=2$ supersymmetric $\sigma$-model with general scalar potential", Nucl. Phys. B233(1984)289;

K. Galicki, "A generalization of the momentum mapping construction for quaternionic Kähler manifolds", Commun. Math. Phys. 108(1987)117;

B. de Wit and A. Van Proeyen, "Special geometry, cubic polynomials and homogeneous quaternionic spaces", Commun. Math. Phys. 149(1992)307, hep-th/9112027; 
L.T. Andrianopoli, M. Bertolini, A. Ceresole, R. D'Auria, S. Ferrara, P. Fré and T. Magri, " $N=2$ supergravity and $N=2$ super Yang-Mills theory on general scalar manifolds:symplectic covariance, gaugings and the momentum map", J. Geom. Phys. 23(1997)111, hep-th/9605032,

B. de Wit, M. Roček and S. Vandoren, "Gauging isometries on hyperkähler cones and quaternion-Kähler manifolds", Phys. Lett. B511(2001)302, hep-th/0104215.

E. Bergshoeff, S. Cucu, T. de Wit, J. Gheerardyn, S. Vandoren and A. Van Proeyen, "The map between conformal hypercomplex/hyper-Kähler and quaternionic(-Kähler) geometry", hep-th/0411209

[8] E. Bergshoeff, S. Cucu, T. de Wit, J. Gheerardyn, R. Halbersma, S. Vandoren and A. Van Proeyen, "Superconformal $N=2, D=5$ matter with and without actions", J. High Energy Phys. 0210(2002)045, hep-th/0205230,

D.V. Alekseevsky and S. Marchiafava, "Quaternionic structures on a manifold and subordinated structures", Ann. Mat. pura appl.(IV)171(1996)205.

[9] D.V. Alekseevsky, V. Cortés, C. Devchand and A. Van Proeyen, "Flows on quaternionic-Kähler and very special manifolds", Commun. Math. Phys. 238(2003)525, hep-th/0109094.

[10] J. Bagger and E. Witten, "Gauge invariant supersymmetric nonlinear sigma model", Phys. Lett. B118(1982)103.

[11] S. Aoyama, "A new visualization of the gauged supersymmetric sigma-model by Killing potentials", Z. Phys. C32(1986)113.

[12] S. Aoyama, "Four-fermi coupling of the supersymmetric non-linear $\sigma$-model on $G / S \otimes$ $\{U(1)\}^{k}$, Nucl. Phys. B578(2000)449, hep-th/0001160.

[13] S. Aoyama and T. Masuda, "The fuzzy $S^{4}$ by quantum deformation", Nucl. Phys. B656(2003)325, hep-th/0212214.

[14] K. Itoh, T. Kugo and H. Kunitomo, "Supersymmetric non-linear realization for arbitrary Kählerian coset space $G / H$ ", Nucl. Phys. B263(1986)386.

[15] S. Coleman, J. Wess and B. Zumino, "Structure of phenomelogical lagrangians. I",Phys. Rev. 177(1969)2239;

C.G. Callan, S. Coleman, J. Wess and B. Zumino, "Structure of phenomelogical lagrangians. II",Phys. Rev. 177(1969)2247. 